\title{
The Design of Constant False Alarm Module Based on CMLD-CFAR Algorithm
}

\author{
Mengjiao $\mathrm{WU}^{1, \mathrm{a}}$,Jun YANG ${ }^{2, \mathrm{~b}}$, Can $\mathrm{ZHAO}^{3, \mathrm{c}}$,Xintao HUANG ${ }^{4, \mathrm{~d}}$ \\ ${ }^{1}$ Department of Computer science and technology, Yunnan University,KunMing,650500,China \\ ${ }^{2}$ Department of Computer science and technology, Yunnan University,KunMing,650500,China \\ ${ }^{3}$ Department of Computer science and technology, Yunnan University,KunMing,650500,China \\ ${ }^{4}$ Department of Computer science and technology, Yunnan University,KunMing,650500,China \\ a email:344786838@qq.com, ${ }^{\text {bemail: junyang@ynu.edu.cn, }{ }^{c} e m a i l: 781831625 @ q q . c o m, ~}{ }^{d}$ email:
} 173471638@qq.com

Keywords: CMLD-CFAR; FPGA; MATLAB; Radar; The Simulation

Abstract.Deleting the average class of constant false alarm rate monitoring (CMLD CFAR), which bases on an average constant false alarm rate monitoring(CA - CFAR) ,can not only overcome the ML type detector under the background of multiple targets detection problem of poor performance, but also address the traditional OS type detector false alarm rate problem of the great losses[1].This paper regards the FPGA as a platform.Firstly use MATLAB simulation produces radar echo data to obey Rayleigh distribution,at the same time,switch this radar echo data into point data,which applies to hardware emulation; And then when using hardware simulation, read the previous data file and deal it with constant false alarm rate processing.At last,the system will show hardware simulation results and waveform.

\section{Introduction}

In recent decades, radar technology has a rapid development,which has been widely applied in the military field and civil field.As for a tool, radar's main task is to obtain the target's position and speed information,and its basic principle is to use the electromagnetic scattering characteristics of objects to detect and identify the target.The rapid development of Modern Electronic Science and Technology provides the opportunity to the progress of radar technology, and the good external environment greatly accelerates the development of radar theory and application technology.

The constant false alarm rate (CFAR) processing technology is an important part of radar signal processing.Radar signal detection is conducted on the interference of background. Interference include thermal noise within the receiver, and the features, such as snow and rain, the sea clutter interference and so on. Among them, the ground clutter, sea clutter, meteorological clutter and chaff clutter, etc are made by antenna beam irradiation area of the scattering signal superposition of scattering unit[2]. To extract the signals in the noise interference, not only requires a certain signal-to-noise ratio, but also needs a CFAR processing equipment. CFAR processing is to keep the purpose of the signal detection of constant false alarm rate, so that the data processing terminal can avoid overload which due to too many false alarm.

\section{The Theoretical Basis}

The purpose of radar's constant false alarm rate processing is to make a target to extract in complex background. Through the threshold as the intensity of the background clutter noise adaptive change,finally maintain a constant false-alarm probability[3]. When the target does not exist, it is only exist background noise or clutter, the detected target probability called the false alarm probability. When there is objective, the probability of detected the target called detection probability. 
Radar for testing to confirm the reflected signal whether there is the identification of each unit. This signal space contains two parts: $s(t)=0$ or $s(t) \neq 0$.Then result in the following two sentences: target signal $s(t)$ does not exist or exist, respectively called $H_{0}$ and $H_{1}$. Discriminate right price will usually thought zero, so it needs to consider the following losses: $s(t)$ does not exist but was found for the existence of loss, represented as $L_{0}$. $s(t)$ existence has sentenced for does not exist the losses, expressed as $L_{1}$. Thus can get the definition of false alarm probability:

$$
P_{f a}=P_{r}[s(t) \text { exist } \mid s(t) \text { not exist }]
$$

Similarly, the definition of detection probability:

$$
P_{d}=P_{r}[s(t) \text { exist } \mid s(t) \text { not exist }]
$$

So, for the average loss:

$$
L=q P_{f a} L_{0}+p\left(1-P_{d}\right) L_{1}
$$

When there are signals of p represents the prior probability, q represents no signal exists prior probability.

\section{The Introduction of CMLD-CFAR}

The adaptive threshold CFAR detection $U_{0}$ is equal to the amount of background noise and clutter intensity $\hat{\mu}$ estimated with a weighted $\mathrm{K}$ product,that is $U_{0}=K \hat{\mu}$. Among them,the weighted quantity $\mathrm{K}$ is only relevant with constant false alarm level and distribution features of the background,but $\hat{\mu}$ associates with the specific detection methods[4]. When adjusting the threshold of multiplier $\mathrm{K}$, can change the size of the threshold $U_{0}$. Under different ways of working to ensure that the detection performance of the optimal, so as to control the size of the false alarm rate. When testing unit $\mathrm{D}$ value is greater than the threshold $U_{0}$, then the signals were ruled as the target goal.According to the calculation of $\hat{\mu}$, CFAR detector is divided into two typical detector-average $(\mathrm{ML})$ and order statistics (OS) , its order statistics (OS) principle block diagram is shown in figure 1 .

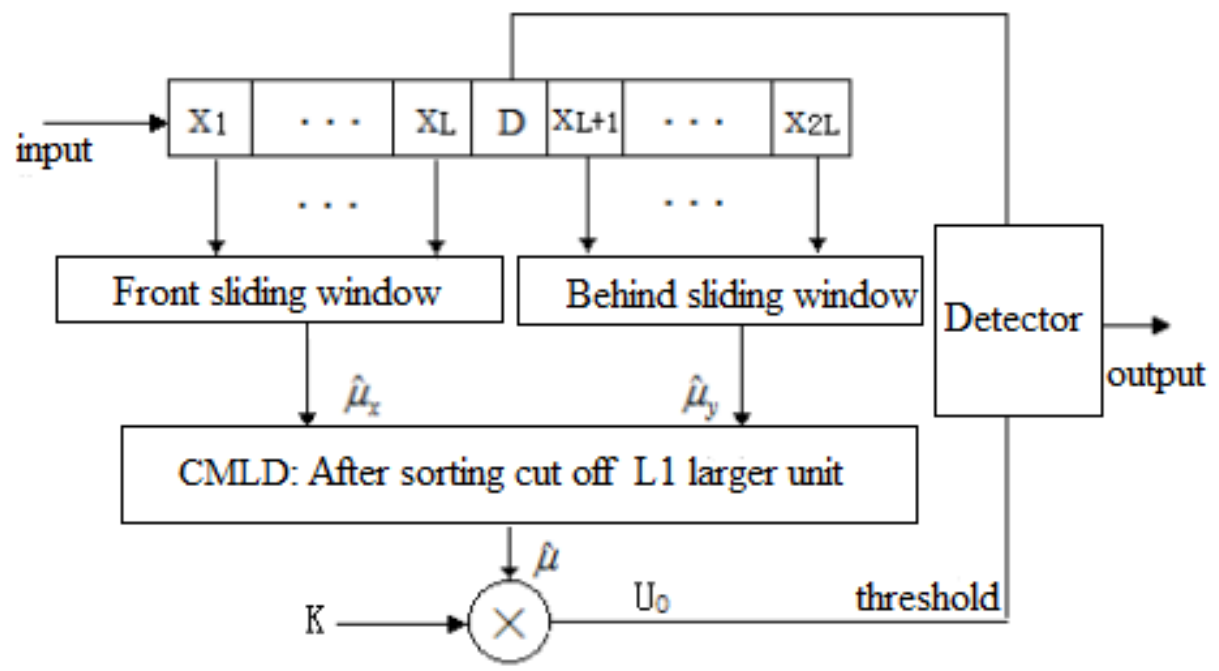

Fig.1. The CMLD-CFAR structure diagram

Input signals are sent to delay line which composed of $(2 L+1)$ delay unit , D is tested unit, D have $\mathrm{L}$ units on each side,which is considered as reference unit. And background clutter and noise energy is through the detection unit $\mathrm{D}$ around $2 \mathrm{~L}$ reference unit for processing. ML CFAR including cell average (CA) CFAR, maximum (GO) CFAR, minimum (SO) CFAR and SO on; OS CFAR including order statistics (OS) CFAR, examination and average level detector (CMLD) CFAR, cut the average (TM) CFAR, etc. Determined by the type of CMLD -CFAR: 
CMLD-CFAR: $\quad \hat{\mu}_{C M L L}=\sum_{i=1}^{2 L-l_{2}} x_{i}$

There: Each unit of the reference signal in the range from small to large order after the first i value expressed $x_{i}$; Since minimum sampling value of $l_{1}$, the smallest unit of reference samples values expressed $x_{l_{1}}$, which in reference to the respective signal sort by range from small to large.Since maximize sampling value of $l_{2}$,the biggest unit of reference samples values expressed $x_{l_{2}}$, which in reference to the respective signal sort by range from small to large[5].

The figure 1 shows that $2 \mathrm{~L}$ reference units calculate average estimate using data window, after each radar transmitted pulse, it will receive all of the echo data from the data window a slip.Due to the limited number of reference unit, the mean estimate there will be some ups and downs. Reference unit is less, the greater the average estimate of ups and downs. In order to maintain the same false alarm rate, it is necessary to raise the threshold adjustment ( $\mathrm{K}$ value). But the increase will reduce detection probability threshold, so need to increase the signal-to-noise ratio found in keeping with the specified

\section{The FPGA implements CMLD - CFAR algorithm}

With the development of radar technology, radar processing algorithm is more and more complex.The amount of data,which needs to been dealt with is becoming bigger and bigger,so the time used to complete the processing task is becoming longer and longer.However,the most important for radar signal processing is the real-time requirements, so the requirement to the processor is becoming more and more rigorous. For complex algorithm,due to the software of radar signal processing and embedded software programming cannot meet the real-time requirements,it requires more efficient processors to deal with radar signal.

In recent years, with the development of the digital circuit technology, FPGA is regarded as a rising signal processor is a hardware programming language.Gradually, the advantages of pure hardware implementation and execution are used in radar signal processing field.For example, the constant false alarm processing, pulse accumulation and so on.The characteristics of these algorithms contains implementing simply,operating simply, and making full use of the FPGA parallelism and the advantages of hardware implementation.

Using FPGA to realize radar clutter constant false alarm rate circuit diagram is shown in figure 2,the basic function mainly includes the following several modules: shift register, adder, multiplier, comparator, for sorting statistics class constant false alarm rate detector, it needs to include sorting and reviewing the module [6].

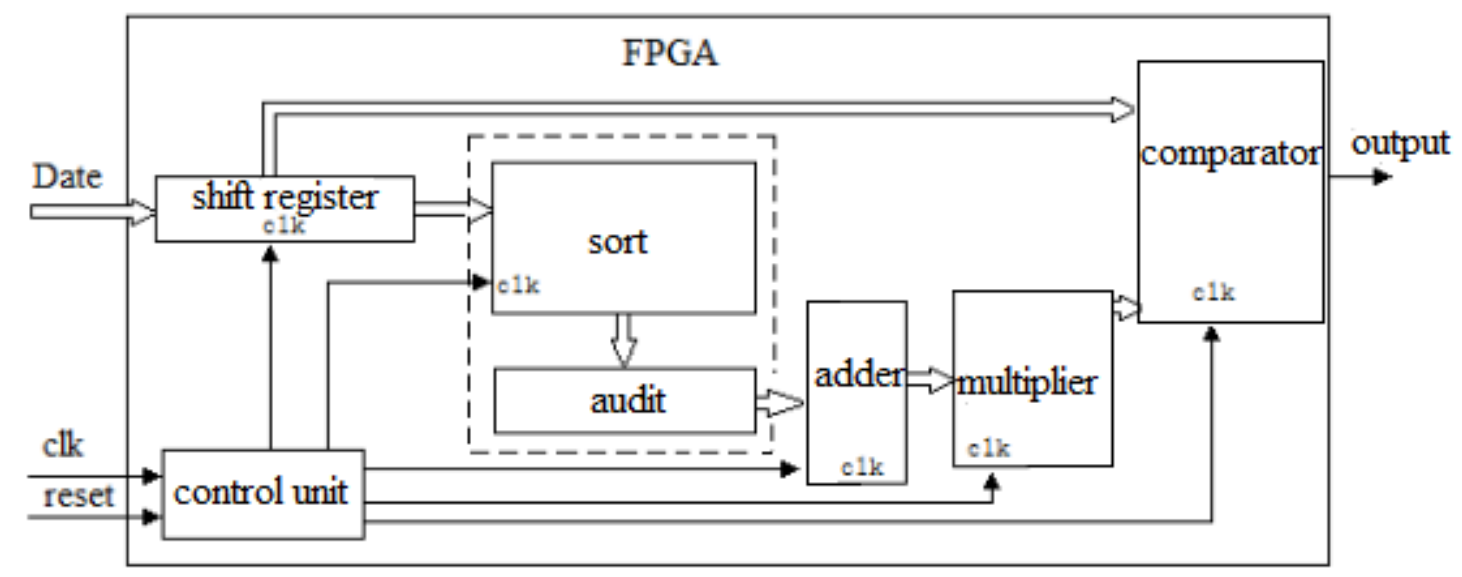

Fig.2.The constant false alarm rate processing block diagram based on th FPGA

MATLAB has many advantages,for example,using easily, programming simply and concise 
language,operation efficiency, abundant content and it's function library is free to expand.What's more,simple drawing, using new data types and object oriented programming technology,it applies to the field of signal analysis and processing. The main task of digital signal processing is to extract, storage, transform,filter,valuate, enhance, compress signals and so on.In the signal processing,MATLAB is mainly used to waveform, spectrum analysis, cepstrum analysis, statistical signal processing, filter design and analysis, etc [7]..

In this work, the radar echo data that comes from the MATLAB should obey Rayleigh distribution then switch this data into a point data,which applies to hardware processor,and as shown in figure 3.Finally,import the produced data into a file. When using hardware simulation, read the previous data file,and deal it with constant false alarm rate processing, then the system will show hardware simulation results and waveform.

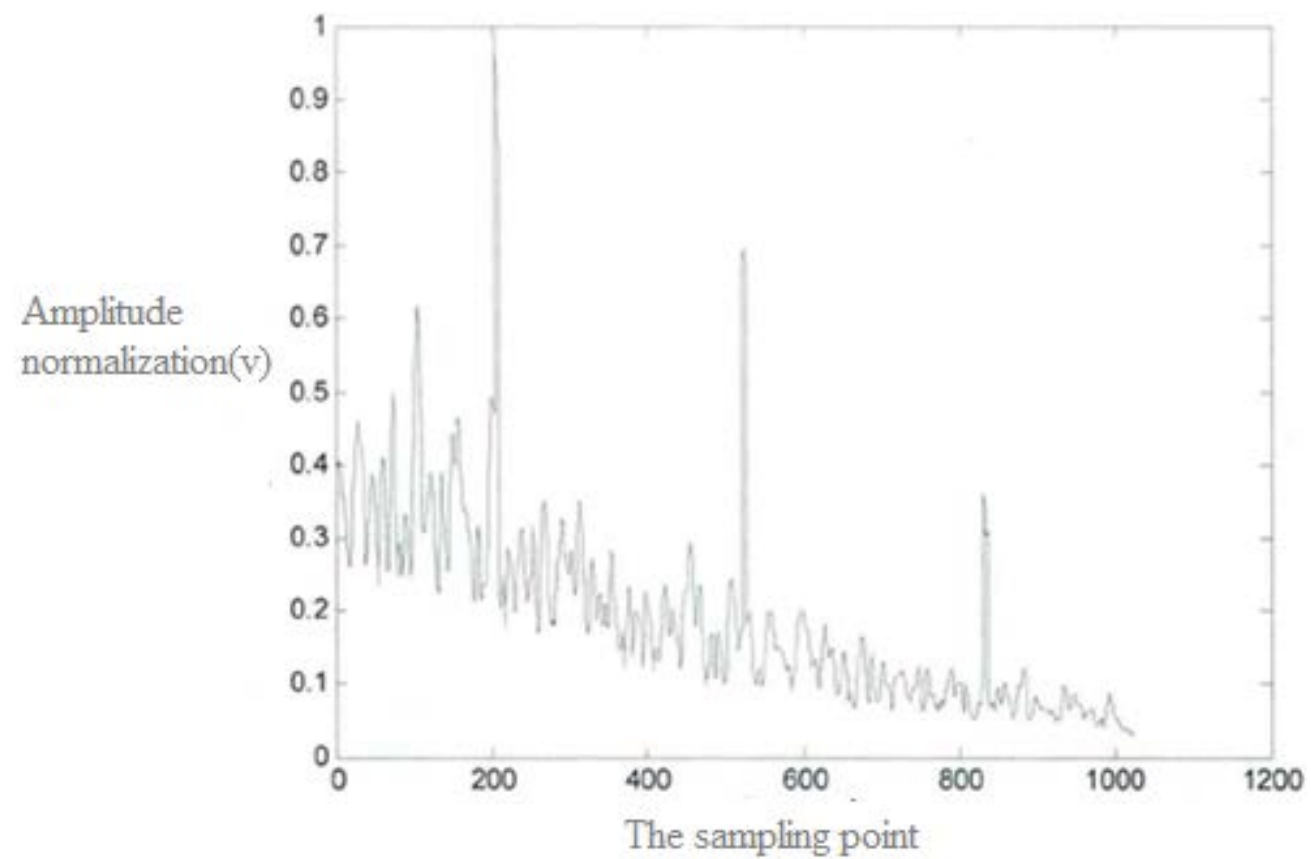

The modelsim simulation

Fig.3.The stack rayleigh distribution clutter of target echo

The results of using FPGA to delete average that belongs to constant false alarm rate algorithm and simulating are shown in figure 4. According to figure 4, din is a original radar echo data,threshold is a limitation that is dealt with CMLD-CFAR,dout is a data,which is outputted from threshold. In the box of figure 4, the radar data (here) "means the target signal" is much higher than the threshold.That is to say the target signal is detected,and output dout,just as the box of figure 4 shows.

According to figure 4,although there is noise interference around the signal to bother it,there is no false target happen.Basically it achieved the purpose of constant false alarm rate detection,.Also it shows that the logical design of FPGA to realize constant false alarm rate detector is correct.
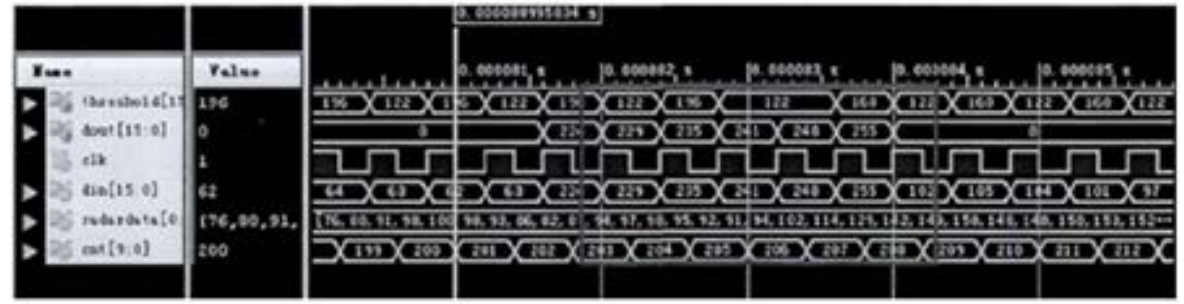

Fig.4.The delet average type of constant false alarm rate processing

\section{Conclusion}

Radar signal of constant false alarm (CFAR) processing is a key link in radar signal 
processing.Firstly,this paper introduces the basic theory of CFAR processing.Secondly, using FPGA to realize and simulate CMLD - CFAR algorithm.Although,CMLD-CFAR algorithm dose well in multitarget and clutter edge cases. On the whole,the overall implementation process is complex,the time to sort it is long and the real-time requirements can't easy to meet.

\section{References}

[1] Xi X.G. Radar clutter of constant false alarm processing research [D]. Dalian maritime university, 2013.

[2] $\mathrm{Xu}$ Y. Based on the FPGA digital radar signal reception processing [D]. Dalian maritime university, 2012.

[3] Yan H.L. Radar signal processing module based on FPGA design [D]. Xi 'an university of electronic science and technology, 2014.

[4] Min J.G. Multi-function radar signal processor based on FPGA [D]. Xi 'an university of electronic science and technology, 2013.

[5]zhao H.T . Radar clutter based on DSP and FPGA is the constant false alarm processing technology research and performance analysis [D]. Dalian maritime university, 2012.

[6] Fan Y.Y Radar signal processing of constant false alarm design implementation [D]. Xi 'an university of electronic science and technology, 2013.

[7] Shao Z.C\&Wang R.D,\&Zhang W . Radar signal processing based on matlab simulation system [J]. Computer simulation, and practices of 2007:268-271. 